Pacific Journal of Mathematic 


\section{A REPRESENTATION OF A BOUNDED FUNCTION AS INFINITE PRODUCT IN A DOMAIN WITH BERGMAN-SHILOV BOUNDARY SURFACE}

\section{SKWARCZYNSKI}

In this paper we consider a closed domain $Q$ in the space of two complex variables along with its decomposition into two parameter family $q\left(\lambda_{1}, \lambda_{2}\right)$ of segments of analytic surfaces. Under some additional assumptions about the domain $Q$ one introduces using Poisson formula the real-valued functions of the real extended class. These functions are harmonic in each $q\left(\lambda_{1}, \lambda_{2}\right)$. This in turn enables us to define the complex valued functions of the complex extended class. The aim of the paper is to show that a bounded analytic function which has infinitely many zero surfaces $n_{s}(z)=0, z=\left(z_{1}, z_{2}\right), s=1,2, \cdots$ can be represented in the domain $Q$ in the form

$$
f(p)=w(p) \prod_{k=1}^{\infty} e_{k}(p), p \in Q, w(p) \neq 0 .
$$

Here $e_{k}(p)$ are functions of the complex extended class.

Potential theoretical methods play a great role in the theory of functions of one complex variable. However, the generalization of these methods to the case of two complex variables is not immediate. The Dirichlet problem for harmonic functions (real parts of analytic functions of one complex variable) has in general a solution. An analog of this result does not hold in the case of two complex variables. In order to overcome this obstacle, Bergman introduces the so-called functions of extended class for a certain tyce of domains with a Bergman-Shilov boundary surface. See [1]. In contrast to harmonic functions these functions depend upon the domain. The problem relating to values prescribed on the Bergman-Shilov boundary of these domains admits a solution for functions of the extended class. One is able to introduce an analog of the Green's function and the generalized Poisson formula. This is done for slightly more general domains in $\S 1-\S 3$. In $\S 4-\S 5$ a further step in this direction is made, namely it is shown that one obtains an analog of the Blaschke product for a bounded analytic function which has infinitely many zero surfaces. Such a function can be represented inside the domain $Q$ as an infinite product of functions of complex extended class with given zero surfaces and the nonvanishing function continuous in $Q$ and analytic on surfaces $q\left(\lambda_{1}, \lambda_{2}\right)$.

In the case of an analytic polyhedron bounded by two segments of analytic hypersurfaces, the functions of extended class have been 
studied by Bergman in [1] and [2]. The analogues of Green's function for this case have been introduced and investigated in [4].

1. The domain $\bar{Q}$ and its representation. We shall assume that the domain $\bar{Q} \subset C^{2}$ is given as a homeomorphic image of the cartesian product of two unit discs $\lambda_{1}^{2}+\lambda_{2}^{2} \leqq 1$ and $|\xi| \leqq 1$.

$$
\boldsymbol{T} \equiv \boldsymbol{T}\left(\lambda_{1}, \lambda_{2}, \xi\right) \equiv\left(T_{1}\left(\lambda_{1}, \lambda_{2}, \xi\right), T_{2}\left(\lambda_{1}, \lambda_{2}, \xi\right)\right)=p \in \bar{Q} .
$$

Further, the homeomorphism $\boldsymbol{T}$ is analytic with respect to the variable $\xi$ and

$$
\left|\frac{\partial T_{1}}{\partial \xi}\right|+\left|\frac{\partial T_{2}}{\partial \xi}\right|>0
$$

$\partial Q=A \cup B$, where $A$ is a segment of an analytic hypersurface

$$
A=\left\{p: p=\boldsymbol{T}\left(\lambda_{1}, \lambda_{2}, \xi\right), \lambda_{1}^{2}+\lambda_{2}^{2}=1\right\}
$$

and

$$
B=\left\{p: p=\boldsymbol{T}\left(\lambda_{1}, \lambda_{2}, \xi\right),|\xi|=1\right\} .
$$

We assume that $B$ is a homeomorphic image of the cartesian product of the unit disc and the unit circle $|\zeta| \leqq 1,0 \leqq \mu \leqq 2 \pi$, respectively. Further, this homeomorphism $t$ is assumed to be analytic with respect to $\zeta$ and

$$
\boldsymbol{t}(\mu, \zeta)=\left(t_{1}(\mu, \zeta), t_{2}(\mu, \zeta)\right)=p \in B,\left|\frac{\partial t_{1}}{\partial \zeta}\right|+\left|\frac{\partial t_{2}}{\partial \zeta}\right|>0 .
$$

Hence $B$ is a segment of an analytic hypersurface. Let $\xi=e^{i \varphi}$, $\varphi \equiv \varphi\left(\lambda_{1}, \lambda_{2}, \mu\right)$. We assume that

$$
\phi^{\prime}=\frac{\partial \varphi\left(\lambda_{1}, \lambda_{2}, \mu\right)}{\partial \mu}
$$

is continuous and different from zero.

Without loss of generality we can require that:

(1) $\left\{\boldsymbol{T}\left(\lambda_{1}, \lambda_{2}, e^{i \varphi}\right): \lambda_{1}=\lambda_{2}=0\right\}=\{\boldsymbol{t}(\mu, \zeta): \zeta=0\}$

(2) $\frac{\partial \varphi(0,0, \mu)}{\partial \mu} \equiv 1$.

The intersection $S=A \cap B$ forms the Bergman-Shilov boundary surface of $Q$. In the following all definitions and theorems pertain to the fixed representation of the domain $\bar{Q}$, in particular to the fixed mappings $\boldsymbol{T}$ and $\boldsymbol{t}$. For briefness we shall write $p=\left[\lambda_{1}, \lambda_{2}, \xi\right]$ instead of $p=$ $\boldsymbol{T}\left(\lambda_{1}, \lambda_{2}, \xi\right)$ and similarly $p=[\mu, \zeta]$ instead of $p=\boldsymbol{t}(\mu, \zeta)$. We shall call the segments of surfaces 


$$
\begin{aligned}
q\left(\lambda_{1}, \lambda_{2}\right) & =\left\{p: p=\left[\lambda_{1}, \lambda_{2}, \xi\right],|\xi| \leqq 1\right\} \\
b(\mu) & =\{p: p=[\mu, \zeta],|\zeta| \leqq 1\}
\end{aligned}
$$

the laminas of $\bar{Q}$ and $B$, respectively. A function defined on the lamina is said to be analytic (harmonic) if it is an analytic (harmonic) function of the variable $\xi$ (or $\zeta$ ) in the disc $|\xi|<1(|\zeta|<1$ ). An analytic (biharmonic) function of two complex variables $\left(z_{1}, z_{2}\right)=z$ defined in $\bar{Q}$ is analytic (harmonic) in each lamina.

2. Functions of REC. Poisson formula and Harnack theorem for regular functions of REC.

Definition. The continuous real function $h(p)$ defined in $\bar{Q}$ belongs to the REC (real extended class) if it is harmonic in each lamina, except perhaps for a finite number of logarithmic poles. If $h(p)$ has no poles (in open laminas), it will be called a regular function of REC.

REMARK. In [1], [2] Bergman has introduced the functions of REC regular in $Q$. He denotes this class by $\mathscr{E}(Q)$.

A function $u(z)$ biharmonic in $Q$ is a regular function of REC. By using the Poisson formula we can express the values $h(p), p \in Q$, of the regular function $h \in \mathrm{REC}$ in terms of its values on $S$, namely for $p=\left[\lambda_{1}, \lambda_{2}, \xi\right]$ we have

$$
h(p)=\frac{1}{4 \pi^{2}} \int_{0}^{2 \pi} \int_{0}^{2 \pi} h\left(\left[\mu, e^{i \not}\right]\right) P_{\xi} P_{\zeta \mathcal{P}^{\prime}} d \mu d \tau^{\prime},
$$

where

$$
\begin{array}{ll}
P_{\xi}=P_{\xi}(\varphi(\mu))=\frac{1-\rho^{2}}{1-2 \rho \cos (\gamma-\varphi(\mu))+\rho^{2}}, & \xi=\rho e^{i \gamma} \\
P_{\zeta}=P_{\zeta}\left(\psi^{\prime}\right)=\frac{1-r^{2}}{1-2 r \cos (\theta-\psi)+r^{2}}, & \zeta=r e^{i \theta} .
\end{array}
$$

The kernel $P_{\xi} P_{\zeta} \varphi^{\prime}$ is a continuous positive function of the variables $p, \mu, \psi, p \in Q$. (See also [2], p. 320.) Hence for each closed subset $K \subset Q$ there exist constants $M_{1}$ and $M_{2}$ such that

$$
0<M_{1} \leqq P_{\xi} P_{\zeta} \varphi^{\prime} \leqq M_{2}, \quad \text { for } p \in K .
$$

The following analog of Harnack's theorem holds:

LEMma. A series of nonnegative regular functions $h_{n} \in \mathrm{REC}$ converges almost uniformly in $Q$ if it converges at one point $p \in Q$. 
The proof follows immediately from inequality (1).

We can also apply this lemma to the functions of the REC considered in the domain

$$
\bar{Q}_{j}=\left\{p: p=\left[\lambda_{1}, \lambda_{2}, \xi\right], \lambda_{1}^{2}+\lambda_{2}^{2} \leqq\left(1-\frac{1}{j}\right)^{2},|\xi| \leqq 1\right\},
$$

for, the restriction of $h \in \mathrm{REC}$ to the domain $\bar{Q}_{j}$ can be regarded, in a natural way, as a function of the REC of the domain $\bar{Q}_{j}$

$$
\operatorname{REC}=\operatorname{REC}(Q) \subset \operatorname{REC}\left(Q_{j}\right) .
$$

3. Green's function of the REC. Let us consider the function $h \in$ REC. Using Poisson's formula, we can define a unique regular function $h_{s} \in$ REC such that $h_{s} \equiv h$ on $S$. The function $G(h, p)=$ $h_{S}(p)-h(p)$ will be called the Green's function of REC with singularity $h$. (See also [1], p. 420.) If $h \leqq 0$, we have the inequality

$$
0 \leqq G(h, p) \leqq-h .
$$

If $h_{1}-h_{2}$ is a regular function of REC, then $G\left(h_{1}, p\right) \equiv G\left(h_{2}, p\right)$. Denote by $G_{\jmath}(h, p)$ the Green's function of $\operatorname{REC}\left(Q_{\jmath}\right)$. Then for $h \leqq 0$,

$$
0 \leqq G_{j}(h, p) \leqq G_{k}(h, p) \leqq G(h, p), \quad \text { for } Q_{j} \subset Q_{k} .
$$

For $h_{1}, h_{2} \in \mathrm{REC}$

$$
G\left(h_{1}+h_{2}, p\right)=G\left(h_{1}, p\right)+G\left(h_{2}, p\right)
$$

holds. If $h(p)$ has no singularities on $S$, then

$$
\lim _{j \rightarrow \infty} G_{\jmath}(h, p)=G(h, p) \text {. }
$$

Let $n_{s}(z)=0, s=1,2, \cdots$, be the equations of analytic surfaces in $\bar{Q}$. We assume that

(1) $\log \left|n_{s}\right| \in$ REC;

(2) $n_{s}(z) \neq 0$ for $z \in S, s=1,2, \cdots$;

(3) surfaces $n_{s}(z)=0$ have no accumulation points on $\bar{Q}-A$. Let us denote

$$
I_{j}=\left\{s: n_{s}(z) \neq 0 \text { for } z \in \bar{Q}_{j}\right\}
$$

4. THEOREM 1. In order that $u(p)$ exists, with almost uniformly convergent representation,

$$
u(p)=\sum_{k=1}^{\infty} h_{k}(p)
$$

such that 
(1') $h_{k}(p) \leqq 0$

(2') $h_{k} \in \mathrm{REC}$

(3') for l large enough, $\sum_{k=1}^{l} h_{k}-\sum_{s \notin I_{j}} \log \left|n_{s}\right|$ is a regular function of the REC $\left(\bar{Q}_{J}\right)$, it is necessary and sufficient that

$$
\sum_{s=1}^{\infty} G\left(\log \left|n_{s}\right|, p\right)<\infty
$$

at one point $p_{0} \in Q$.

Proof. Necessity: We shall show that the partial sums of (4) are bounded at some points of $Q_{j}$. From condition (3) follows that for fixed $m$ and $j$ large enough,

$$
\begin{aligned}
\sum_{s=1}^{m} G_{j}\left(\log \left|n_{s}\right|, p\right) & \leqq \sum_{s \notin I_{j}} G_{j}\left(\log \left|n_{s}\right|, p\right)=G_{j}\left(\sum_{s \notin I_{j}} \log \left|n_{s}\right|, p\right) \\
& =G_{j}\left(\sum_{k=1}^{l} h_{k}, p\right) \leqq-\sum_{k=1}^{l} h_{k}(p) \leqq-u(p) .
\end{aligned}
$$

Since $\log \left|n_{s}(z)\right|$ has no singularities on $S$,

$$
\sum_{s=1}^{m} G\left(\log \left|n_{s}\right|, p\right)=\lim _{j \rightarrow \infty} \sum_{s=1}^{m} G_{j}\left(\log \left|n_{s}\right|, p\right) \leqq-u(p) .
$$

Hence series (4) converges at points $p$ for which $-u(p)<\infty$. From condition (3) follows that there are points $p \in Q_{j}$ for which $n_{s}(p) \neq 0$, $s=1,2, \cdots$. For these points $\left(3^{\prime}\right)$ yields $-h_{k}(p)<\infty, k=1,2, \cdots$. Hence $-u(p)<\infty$.

Sufficiency: Let $h_{k}(p)=G\left(\log \left|n_{k}\right|, p\right)$. The conditions $\left(1^{\prime}\right),\left(2^{\prime}\right),\left(3^{\prime}\right)$ hold for functions $h_{k}(p)$. Let $K$ be a closed subset of $Q$ and let $K \subset Q_{j}, P_{0} \in Q_{j}$. For large enough $k$ the functions $h_{k}$ are regular of the REC $\left(Q_{j}\right)$. Since series (4) converges in the point $p_{0} \in Q_{j}$, its almost uniformly convergence on $Q_{j}$ follows from the lemma. Hence it converges uniformly on $K$ and we can define

$$
u(p)=\sum_{k=1}^{\infty} h_{k}(p)
$$

In order to formulate an analog of Theorem 1 for complex functions, we introduce the following:

Definition. A function $e(p)$ continuous in $\bar{Q}$ and analytic in each $q\left(\lambda_{1}, \lambda_{2}\right)$ is said to belong to the CEC (complex extended class) if $\log |e(p)| \in$ REC.

5. THEOREM 2. In order that $f(p)$ exists with an almost uniformly convergent representation 


$$
f(p)=\prod_{k=1}^{\infty} e_{k}(p)
$$

such that

(1') $\left|e_{k}(p)\right| \leqq 1$,

(2') $e_{k}(p) \in \mathrm{CEC}$,

(3') for l large enough $\sum_{k=1}^{l} \log \left|e_{k}(p)\right|-\sum_{s \notin I_{j}} \log \left|n_{s}(p)\right|$ is a regular function of the $\operatorname{REC}\left(Q_{j}\right)$,

it is necessary and sufficient that $\sum_{s=1}^{\infty} G\left(\log \left|n_{s}\right|, p\right)<\infty$ at one point $p_{0} \in Q$.

Proof. Necessity: We have

$$
\log |f(p)|=\sum_{k=1}^{\infty} \log \left|e_{k}(p)\right| .
$$

The convergence of (4) follows from Theorem 1.

Sufficiency: $\left.\quad G\left(\log \left|n_{s}\right|, p\right)=\log \left|n_{s}\right|\right)_{S}-\log \left|n_{s}(p)\right|$. Let $\nu_{s}$ denote a function which in each lamina $q\left(\lambda_{1}, \lambda_{2}\right)$ is conjugate to $\left(\log \left|n_{s}\right|\right)_{S}$ and vanishes at $p=\left[\lambda_{1}, \lambda_{2}, 0\right]$. Let $r_{s}$ be the radius of the largest disc such that $n_{s}(p) \neq 0$ for $p=\left[\lambda_{1}, \lambda_{2}, \xi\right], \lambda_{1}^{2}+\lambda_{2}^{2}<r_{s}^{2}$. Let $\kappa_{s}(r)$ denote the continuous function defined as follows: for $r_{s} \neq 0$,

$$
\kappa_{s}(r)=\left\{\begin{array}{cl}
0 & 1 \\
\text { linear for } & r_{s}^{2} \geqq r \geqq r \geqq r_{s}^{2} \\
1 & r_{s}^{3} \geqq r \geqq 0
\end{array},\right.
$$

and for $r_{s}=0, \kappa_{s}(r) \equiv 0$. Let us consider the function $\arg \left(n_{s}(p)\right)$. This function can be regarded as univalent in the simply-connected domain $\left\{p=\left[\lambda_{1}, \lambda_{2}, \xi\right]: \lambda_{1}^{2}+\lambda_{2}^{2} \leqq\left(r_{s}^{2}\right)^{2}\right\}$. Define

$$
\begin{array}{rlr}
c_{s} & =\nu_{s}-\left(\arg n_{s}-\kappa_{s}(r) \arg n_{s}\left(\left[\lambda_{1}, \lambda_{2}, 0\right]\right)\right), \quad r^{2}=\lambda_{1}^{2}+\lambda_{2}^{2} \\
e_{k}(p) & =\exp \left(G\left(\log \left|n_{k}\right|, p\right)+i c_{k}(p)\right) .
\end{array}
$$

The conditions $\left(1^{\prime}\right),\left(2^{\prime}\right),\left(3^{\prime}\right)$ of the theorem hold for functions $e_{k}(p)$. The series $\sum_{k=1}^{\infty} G\left(\log \left|n_{k}\right|, p+i c_{k}(p)\right.$ converges almost uniformly in $Q$. For, if $k$ is large enough, the function $e_{k}(p)$ is conjugate to $G\left(\log \left|n_{k}\right|, p\right)$ on each lamina $q\left(\lambda_{1}, \lambda_{2}\right) \subset \bar{Q}_{j}$ and $c_{k}\left(\left[\lambda_{1}, \lambda_{2}, 0\right]\right)=0$. Hence the series $\sum_{k=1}^{\infty} c_{k}(p)$ converges almost uniformly on $Q_{j}$ simultaneously with $\sum_{k=1}^{\infty} G\left(\log \left|n_{k}\right|, p\right)$. Consequently, the product

$$
f(p)=\prod_{k=1}^{\infty} e_{k}(p)
$$

converges almost uniformly in $Q$.

CoRollary. Let $f(z)$ be a bounded analytic function defined in 
$\bar{Q}-A$. Let $n_{s}(z)=0$ be the zero surfaces of $f$, i.e., for each $\bar{Q}_{j}$ let

$$
\log |f|-\sum_{s \notin I_{j}} \log \left|n_{s}\right|
$$

be regular in $Q$. Then $f(z)$ can be represented in the form of an almost uniformly convergent product

$$
f(p)=w(p) \prod_{k=1}^{\infty} e_{k}(p), \quad p \in Q,
$$

where $e_{k}$ are given by (5). Here $\log \left|e_{k}(p)\right|=G\left(\log \left|n_{k}\right|, p\right)$. The conditions $\left(1^{\prime}\right),\left(2^{\prime}\right),\left(3^{\prime}\right)$ of Theorem 2 are fulfilled and $w(p)$ is continuous, different from zero and analytic in each lamina $q\left(\lambda_{1}, \lambda_{2}\right) \subset Q$.

Proof. Without loss of generality we may assume that $|f(p)| \leqq 1$. The representation

$$
f(p)=\prod_{k=1}^{\infty} f_{k}(p),
$$

where $f_{1}(p)=f(z), f_{k}(p) \equiv 1$, for $k>1$, satisfy the conditions of Theorem 2. Let us consider the function

$$
g(p)=\prod_{k=1}^{\infty} e_{k}(p)
$$

Setting $w(p)=f(p) / g(p)$, we obtain (6).

\section{BIBLIOGRAPHY}

1. S. Bergman, On zero and pole surfaces of functions of two complex variables, Trans. Amer. Math. Soc. 77 (1954), 413-454.

2. - Über meromorphe Funktionen von zwei komplexen Veränderlichen, Comp. Math. 6 (1939), 305-335.

3. S. Bergman and J. Marcinkiewicz, Sur les fonctions analytiques de deux variables complexes, Journal of Math. and Physics 21 (1942), 125-141.

4. S. Bergman and M. Schiffer, Potential theoretic methods in the theory of functions of two complex variables, Comp. Math. 10 (1952), 213-240.

5. R. Nevanlinna, Eindeutige analytische Funktionen, Berlin, 1936.

Received March 23, 1967. This work was supported in part by NSF grant GP 5797 and AF grant AF-1047-66 at Stanford University.

STANFORD UNIVERSITY 



\section{PACIFIC JOURNAL OF MATHEMATICS}

\section{EDITORS}

H. ROYDEN

Stanford University

Stanford, California

\section{J. P. Jans}

University of Washington

Seattle, Washington 98105

\section{J. DUGUNDJI}

Department of Mathematics University of Southern California Los Angeles, California 90007

\section{RICHARD ARENS}

University of California

Los Angeles, California 90024

\section{ASSOCIATE EDITORS}

E. F. BECKENBACH

B. H. NEUManN

F. WOLF

K. YosIDA

\section{SUPPORTING INSTITUTIONS}

UNIVERSITY OF BRITISH COLUMBIA CALIFORNIA INSTITUTE OF TECHNOLOGY UNIVERSITY OF CALIFORNIA MONTANA STATE UNIVERSITY UNIVERSITY OF NEVADA NEW MEXICO STATE UNIVERSITY OREGON STATE UNIVERSITY UNIVERSITY OF OREGON OSAKA UNIVERSITY UNIVERSITY OF SOUTHERN CALIFORNIA
STANFORD UNIVERSITY

UNIVERSITY OF TOKYO UNIVERSITY OF UTAH WASHINGTON STATE UNIVERSITY UNIVERSITY OF WASHINGTON AMERICAN MATHEMATICAL SOCIETY CHEVRON RESEARCH CORPORATION TRW SYSTEMS NAVAL WEAPONS CENTER 


\section{Pacific Journal of Mathematics}

\section{Vol. 25, No. $1 \quad$ September, 1968}

Glen Eugene Bredon, Cosheaves and homology................... 1

Robin Ward Chaney, A chain rule for the transformation of integrals in

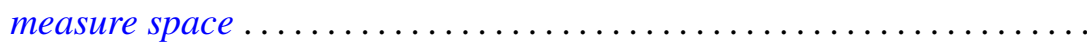

Colin W. Clark, On relatively bounded perturbations of ordinary differential operators................................... 59

John Edwin Diem, A radical for lattice-ordered rings.............. 71

Zeev Ditzian, On a class of convolution transforms ................ 83

Dennis Garoutte and Paul Adrian Nickel, A note on extremal properties characterizing weakly $\lambda$-valent principal functions............. 109

Shwu-Yeng Tzeng Lin, Fixed point properties and inverse limit spaces . . . 117

John S. Lowndes, Some dual series equations involving Laguerre polynomials ................................. 123

Kirti K. Oberai, Sum and product of commuting spectral operators ....... 129

J. N. Pandey and Armen H. Zemanian, Complex inversion for the generalized convolution transformation..................... 147

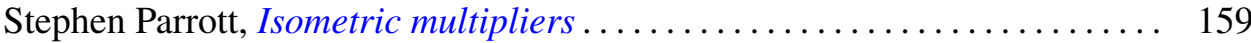

Manoranjan Prasad, Note on an extreme form .................. 167

Maciej Skwarczyński, A representation of a bounded function as infinite product in a domain with Bergman-Shilov boundary surface ......... 177

John C. Taylor, The Šilov boundary for a lattice-ordered semigroup ...... 185 Donald Reginald Traylor and James Newton Younglove, On normality and pointwise paracompactness ............................. 193

L. Tzafriri, Quasi-similarity for spectral operators on Banach spaces ... 